\title{
Carcinosarcoma of the Gallbladder with Two Heterologous Components: A Case Report and Review of the Literature
}

EL MEHDI TIABI ( $\nabla$ tiabi.elmehdi@gmail.com )

Mohammed I University Oujda Faculty of Medicine and Pharmacy Oujda: Universite Mohammed Premier Oujda Faculte de Medecine et de Pharmacie Oujda https://orcid.org/0000-0003-4875-4816 ACHRAF MIRY

Mohammed I University Oujda Faculty of Medicine and Pharmacy Oujda: Universite Mohammed Premier Oujda Faculte de Medecine et de Pharmacie Oujda

\section{ANASS HALOUI}

Mohammed I University Oujda Faculty of Medicine and Pharmacy Oujda: Universite Mohammed Premier Oujda Faculte de Medecine et de Pharmacie Oujda

\section{YOUNESSE NAJIOUI}

Mohammed I University Oujda Faculty of Medicine and Pharmacy Oujda: Universite Mohammed Premier Oujda Faculte de Medecine et de Pharmacie Oujda

\section{MOHAMED BOUDOU}

Mohammed I University Oujda Faculty of Medicine and Pharmacy Oujda: Universite Mohammed Premier Oujda Faculte de Medecine et de Pharmacie Oujda

\section{NASSIRA KARICH}

Mohammed I University Oujda Faculty of Medicine and Pharmacy Oujda: Universite Mohammed Premier Oujda Faculte de Medecine et de Pharmacie Oujda

\section{MOHAMMED BOUZIANE}

Mohammed I University Oujda Faculty of Medicine and Pharmacy Oujda: Universite Mohammed Premier Oujda Faculte de Medecine et de Pharmacie Oujda

\section{AMAL BENNANI}

Mohammed I University Oujda Faculty of Medicine and Pharmacy Oujda: Universite Mohammed Premier Oujda Faculte de Medecine et de Pharmacie Oujda

\section{Case Report}

Keywords: Carcinosarcoma, gallbladder, treatment, prognosis

Posted Date: November 24th, 2020

DOl: https://doi.org/10.21203/rs.3.rs-112928/v1 
License: (c) (i) This work is licensed under a Creative Commons Attribution 4.0 International License. Read Full License 


\section{Abstract}

\section{Introduction}

Gallbladder carcinosarcoma [CSGB] is a rare malignant tumor characterized by malignant epithelial and mesenchymal components. Its pathogenesis is unknown and most CSGBs are associated with poor survival because the disease normally presents at an advanced stage and, therefore, curative resection is rare.

\section{Case report}

This report describes the case of a 66-year-old man with pain in the upper right quadrant. The preoperative diagnosis was cancer of the gallbladder [GB], and therefore, a curative radical cholecystectomy was performed. However, pathological examination of the surgical sample revealed that the tumor was made up of two histological components. The first one is the carcinomatous component and the second one corresponds to the sarcomatous component, which was compatible with a diagnosis of carcinosarcoma.

\section{Conclusion}

The prognosis for CSGB remains poor despite curative resection, and therefore, the authors recommend that efforts be made to improve surgical outcomes.

\section{Background}

Carcinosarcomas are rare tumors that are characterized by malignant epithelial and mesenchymal elements. These tumors have been reported in many different organs, including the uterus, lung, esophagus, kidney, and pancreas [1-3]. Carcinosarcomas of the gallbladder are uncommon. Up to now, less than 100 cases have been reported in the English literature. The invasive nature and aggressive biology of carcinosarcoma of gallbladder adequately explains the limited number of respectable cases. We report a case of carcinosarcoma of gallbladder treated by curative radical cholecystectomy.

\section{Case Presentation}

66 year old patient, who has a history of active chronic smoking at the rate of 40 packs / year, and peritonitis by perforation of a gastric ulcer 30 years ago, who consults for pain in the level of the right hypochondrium, radiating towards the scapula and the right shoulder, associated with bilious vomiting, without any other associated sign, evolving in a context of apyrexia and preservation of the general state. The pain appeared 6 months before his admission. On clinical examination, we find a slight sensitivity in the right hypochondrium, defenseless. The rest of the exam is unremarkable. The patient underwent an abdominal CT scan which was in favor of a distended gallbladder, with the presence of an intramural tissue formation of suspicious appearance, measuring $11 \mathrm{~cm}$, without deep lymphadenopathy. [Fig. 1]. 
He had a resection of the gallbladder with a bisegmentectomy of the liver. [Figure2].

The macroscopic study identified a cholecystectomy with hepatic bisegmentectomy. The gallbladder was $16 \mathrm{~cm}$ long and $10 \mathrm{~cm}$ in diameter. It was a tumor of $15 \times 6 \mathrm{~cm}$, which spread over almost the entire gallbladder and filled the entire gallblader lumen. The mass was brittle reddish-red in color, with hemorrhagic changes. No gallstones were identified. On the section, the tumor extend to the surrounding tissues, reaching the duodenal and hepatic border. [Figure 3].

-Histologically, it is a malignant tumor proliferation, of biphasic appearance, made of a mixture of a component carcinomatous and sarcomatous. The carcinomatous camposante is mainly represented by glands, made of medium to large cells, with abundant eosinophilic cytoplasm. The nuclei are atypical, marked, nucleolated, with chromatin sometimes clear sometimes dense, associated with high number of mitotic foci.

The sarcomatous component is made of a proliferation largely necrotic. The viable tumor is mainly arranged in fascicles. It is made of tumor cells, comprising a spindle-shaped or rounded nucleus, with regular chromatin, finely nucleated, with an abundant and clear cytoplasm. In places, the saromatous component describes vascular cracks, associated with a double heterologous component, describing in some places angiosarcoma, and in others, striated muscle.

No vascular emboli or peri-nervous sheaths have been seen.

Presence of extensive tumor necrosis.

The cystic and duodenal boundaries were in contact with the tumor. The parietal and hepatic resection limits were healthy. [Figure 4].

-An immunohistochemical study was carried out, and objectified:

Positive labeling of tumor cells by CD31 and CD34 in areas of vascular differentiation.

A positive labeling of tumor cells by desmin, in the zones of striated muscle differentiation.

Weak positive labeling with AML and CK7.[Figure 5], [Figure 6], [Figure7].

\section{Discussion And Conclusion}

The first case of a carcinosarcoma of the gallbladder was reported by Landsteiner in 1907 [4]. Up to now, $<100$ cases have been reported in the English literature. Carcinosarcomas of the gallbladder consist of both epithelial and mesenchymal components. The diagnosis requires the presence the two histological components. The epithelial component usually consists of adenocarcinoma, although an element of squamous cell carcinoma is also occasionally observed. The mesenchymal component typically consists of undifferentiated spindle or satellite cells, and is occasionally accompanied by various proportions of heterogenous elements, including chondrosarcoma, osteosarcoma, rhabdomyosarcoma and 
leiomyosarcoma [5]. Zhang et al [6] reported that adenocarcinoma was the most common epithelial component [79.2\%] while squamous cell carcinoma was the least common [9.4\%]. Spindle cell type was the most common mesenchymal component [44.6\%] when compared with osteoid, which was the least common [5.4\%].

Although recent studies suggest some causative factors, such as multistep genetic alterations, the precise mechanisms of gallbladder carcinogenesis have not been clearly elucidated. At present, two opposing theories have been hypothesized to account for the origin of these morphologically diverse tumors. The multiclonal theory regards a carcinosarcoma as a collision tumor composed of the derivatives of two or more stem cells of separate epithelial and mesenchymal origin. The monoclonal theory proposes that carcinomatous and sarcomatous elements are derived from a single pluripotential stem cell that subsequently develops divergent differentiation along separate epithelial and mesenchymal pathways [7]. Dacic et al [8] found identical allelic losses shared by each tumor component, without discordant losses. This was consistent with the hypothesis that the carcinomatous and sarcomatous components of this neoplasm were derived from a single pluripotent stem cell and that the tumor was monoclonal.

GB cancers account for 1 to $2 \%$ of all cancers. CSGB is a rare malignant neoplasm representing less than $1 \%$ of all gallbladder malignant tumors [2], characterized by both malignant epithelial and mesenchymal components with a poorer prognosis than ordinary gallbladder adenocarcinoma. A meta-analysis study of 68 cases of CSGB showed the mean age of the patients was 68.8 years. There were 16 males and 52 females, with male to female ratio of $1 / 3.25$. The mean survival was 17.5 months, with a median of 5 months, and a range between 0 and 85 months [6].

The symptoms and signs of gallbladder carcinosarcoma are non-specific. Representative clinical symptoms include right upper abdominal pain and masses, loss of appetite, weight loss, general fatigue, jaundice, and vomiting. In addition, $66.7 \%-83 \%[9,10]$ of the cases presented with simultaneous cholecystolithiasis. Our patient consults for pain in the level of the right hypochondrium, radiating towards the scapula and the right shoulder, associated with bilious vomiting, without any other associated sign, evolving in a context of apyrexia and preservation of the general state.

The macroscopic study shows that our patient had a tumor that filled the lumen measuring $15 \mathrm{~cm}$ in maximum dimension. In a review of 41 patients with CSGB who had data on tumor size and survival, it was demonstrated that patients with tumors measuring $\geq 5 \mathrm{~cm}$ had a significantly shorter survival when compared with patients who had tumors measuring less than $5 \mathrm{~cm}$. [6].

Gallstones were observed in 14 out of 18 cases in one review $77 \%$, and in $66.7 \%$ of cases in a another one [6]. This high association between gallstones and CSGB is similar to what is observed with carcinoma of the gallbladder. Our patient did not have gallstones found in the lumen of the gallbladder.

The diagnosis of CSGB must be confirmed by microscopic results and immunohistochemical staining. Under the microscope, the diagnosis of CSGB requires the presence of malignant and mesenchymal 
epithelial elements, although these can be intimately mixed and present intermediate characteristics between the two types of cells.

The most common epithelial component is adenocarcinoma, but an epidermoid carcinoma component is also frequently present. In addition, squamous cell carcinoma is known to develop at twice the speed of adenocarcinomas and have a less favorable prognosis [7]. The most common mesenchymal component is spindle cell sarcoma.

Immunohistochemically, the carcinomatous component is positive for epithelial markers, such as cytokeratin and epithelial membrane antigen [EMA], and the sarcomatoid component is positive for mesenchymal markers such as vimentin, desmin, CD31, CD34, and SMA.

For our patient, microscopic examination shows a malignant tumor proliferation, of biphasic appearance, made of a mixture of a component carcinomatous and sarcomatous. The carcinomatous camposante is mainly represented by glands, made of medium to large cells, with abundant eosinophilic cytoplasm. the nuclei are atypical, marked, nucleolated, with chromatin sometimes clear sometimes dense.

The sarcomatous component is made of a proliferation largely necrotic. The viable tumor is mainly arranged in fascicles. It is made of tumor cells, comprising a spindle-shaped or rounded nucleus, with regular chromatin, finely nucleated, with an abundant and clear cytoplasm. In places, the saromatous component describes vascular cracks.

The immunohistochemical study objectified a positive labeling of tumor cells by CD31 and CD34 in areas of vascular differentiation, and a positive labeling of tumor cells by desmin, in the zones of striated muscle differentiation. The IHC shows also a weak positive labeling with AML and CK7.

Zhang et al [6] reported that tumors which were smaller than $5 \mathrm{~cm}$ had a longer survival and that therefore, the tumor size should be considered as a major component in the future staging system. They also indicated that the presence of gallstones, epithelial and mesenchymal component types, age and sex were of little prognostic value.

The most common treatments are surgery. There is no successful treatment reported with radiotherapy or chemotherapy [11].

The prognosis of this disease is normally poor. Most cases present with locally advanced disease. Liver metastasis and peritoneal dissemination are widespread in these cases. Adrenal glands, pancreas, diaphragm, and lower thoracic vertebrae are the other metastatic sites reported in the literature. Regional, retroperitoneal, and para-aortic lymph nodes may be involved. The mean survival time after diagnosis is usually only a few months [12-13]. We analyzed data from 20 patients recorded in the literature from 1980 to 2013 who underwent surgical management for carcinosarcoma of the gall bladder. These patients consisted of 8 male and 12 female with a mean age of 65.2 years (range 45 to 83 years). Zhang et al [6] reported that for 68 patients with carcinosarcoma of the gallbladder, the mean survival time is 17.5 months, and the 1-year survival rate and 5-year survival rate are $(19 \pm 5) \%$ and $(16 \pm 5) \%$ respectively. 
As reported by Uzun MA, the longest survival time was 54 months [14]. In that case, a polypoid tumor of $2 \mathrm{~cm}$ in size protruded into the gallbladder lumen. A microscopic study showed that the tumor had infiltrated the surrounding connective and adipose tissue overlapping its primary site, but had not perforated the serosa nor invaded the liver. The patient was handled by a radical cholecystectomy and remained healthy for 54 months.

CSGB is a rare malignant neoplasm representing less than $1 \%$ of all gallbladder malignant tumors. The most common treatments are surgery. The prognosis of this disease remain poor.

\section{Abbreviations}

GB: Gallbladder

GSGB: Gallbladder carcinosarcoma

CT: Computerized tomography

\section{Declarations}

- Ethics approval and consent to participate:

Not applicable.

- Consent for publication:

A signed consent is obtained from the patient.

- Availability of data and materials:

The datasets used and/or analysed during the current study are available from the corresponding author on reasonable request.

\section{- Competing interest:}

The authors declare that they have no competing interests

- Funding:

Not applicable

- Authors' contributions:

All authors read and approved the final manuscript.

- Acknowledgements: 


\section{References}

1. Iwaya T, Maesawa C, Tamura G, Sato N, Ikeda K, Sasaki A, Othuka K, Ishida K, Saito K, Satodate R. Esophageal carcinosarcoma: a genetic analysis. Gastroenterology. 1997;113:973937. [PubMed] [Google Scholar].

2. J. Albores-Saavedra, D. E. Henson, and D. S. Klimstra, "Benign and malignant mesenchymal, nonepithelial, and neurogenic tumors. 'Miscellaneous Malignant Tumors. Carcinosarcoma'," in Tumors of the Gallbladder, Extrahepatic Bile Ducts, and Vaterian System. AFIP Atlas of Tumor Pathology. Series 4. Fascicle 23, Chapter 7, pp. 202-205, American Registry of Pathology, Silver Spring, MD, USA, 2015.View at: Google Scholar.

3. Reuter VE. Sarcomatoid lesions of the urogenital tract. Semin Diagn Pathol. 1993;10:188201. [PubMed] [Google Scholar].

4. Landsteiner K. Plattenepithelkarzinom und Sarkom der Gallenblasse in einem falle von Gallenblasse. Ztschr Klin Med. 1907;62:427-433. [Google Scholar].

5. Kuroki T, Tajima Y, Matsuo K, Kanematsu T. Genetic alterations in gallbladder carcinoma. Surg Today. 2005;35:101-105. [PubMed] [Google Scholar].

6. Zhang L, Chen Z, Fukuma M, Lee LY, Wu M. Prognostic Signifiance of Race and Tumor Size in Carcinosarcoma of Gallbladder: a Meta-Analysis of 68 Cases. Int J Clin Exp Pathol. 2008;1:7583. [PMC free article] [PubMed] [Google Scholar].

7. Uzun MA, Koksal N, Gunerhan Y, Celik A, Guneş P. Carcinosarcoma of the gallbladder: report of a case. Surg Today. 2009;39:168-171. [PubMed] [Google Scholar].

8. Dacic S, Finkelstein SD, Sasatomi E, Swalsky PA, Yousem SA. Molecular pathogenesis of pulmonary carcinosarcoma as determined by microdissection-based allelotyping. Am J Surg Pathol. 2002;26:510-516. [PubMed] [Google Scholar].

9. Lazcano-Ponce EC, Miquel JF, Munoz N, Herrero R, Ferrecio C, Wistuba II, Alonso de Ruiz P, Aristi Urista G, Nervi F. Epidemiology and molecular pathology of gallbladder cancer. CA Cancer J Clin. 2001;51:349-364. [PubMed] [Google Scholar].

10. Nimura Y. Extended surgery in bilio-pancreatic cancer: The Japanese experience. Semin Oncol. 2002;29:17-22. [PubMed] [Google Scholar].

11. Sodergren MH, Silva MA, Read-Jones SL, Hubscher SG, Mirza DF. Carcinosarcoma of the biliary tract: two case reports and a review of the literature. Eur J Gastroenterol Hepatol. 2005;17:683685. [PubMed] [Google Scholar].

12. Hotta T, Tanimura H, Yokoyama S, Ura K, Yamaue H. So-called carcinosarcoma of the gallbladder; spindle cell carcinoma of the gallbladder: report of a case. Surg Today. 2002;32:462467. [PubMed] [Google Scholar]. 
13. lezzoni JC, Mills SE. Sarcomatoid carcinomas (carcinosarcomas) of the gastrointestinal tract: a review. Semin Diagn Pathol. 1993;10:176-187. [PubMed] [Google Scholar]

14. Mansori KS, Cho SY. Malignant mixed tumor of the gallbladder. Am J Clin Pathol. 1980;73:709711. [PubMed] [Google Scholar].

\section{Figures}

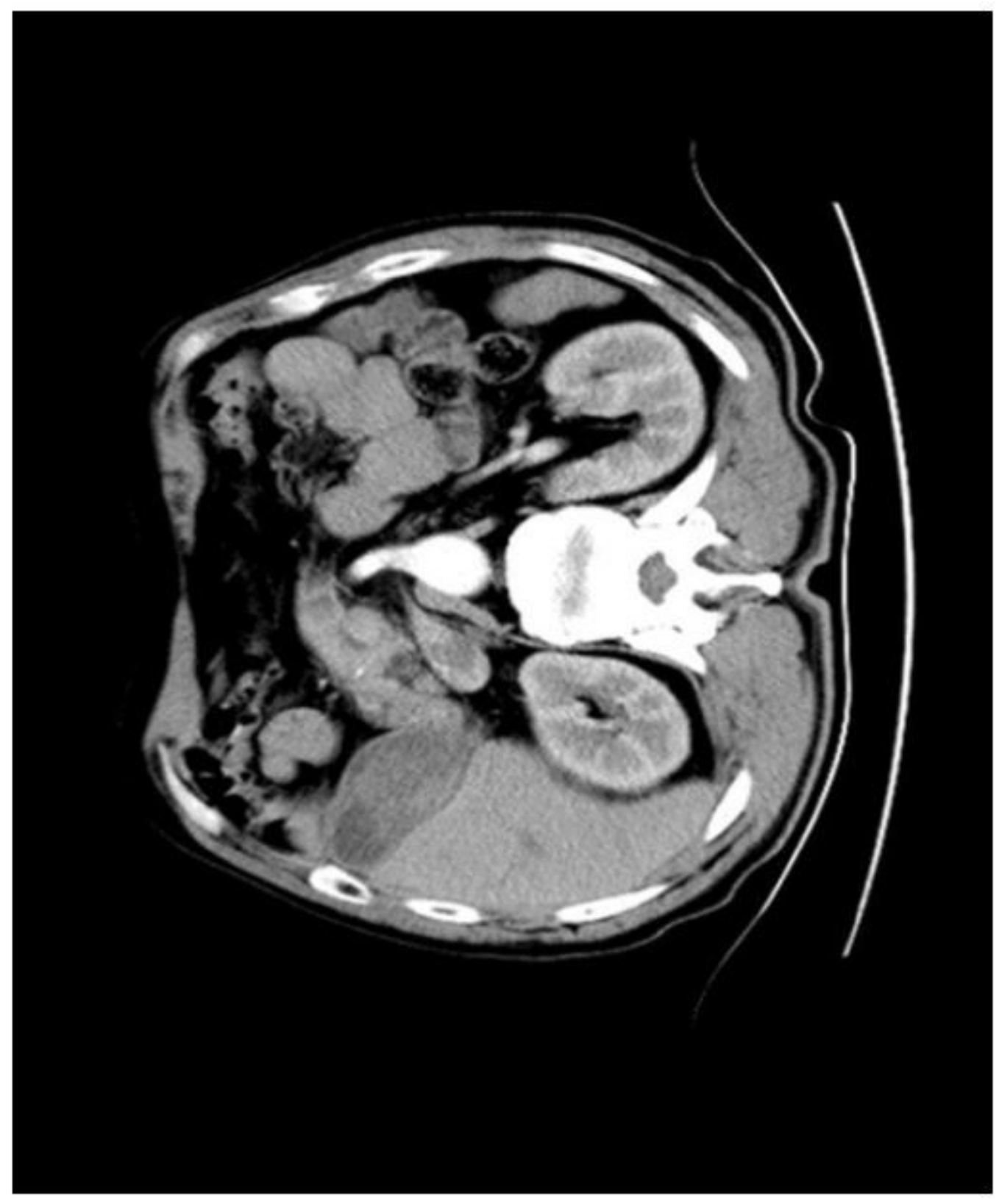

\section{Figure 1}

Abdomen computed tomography showed an intraluminal masse possible gallbladder cancer. 


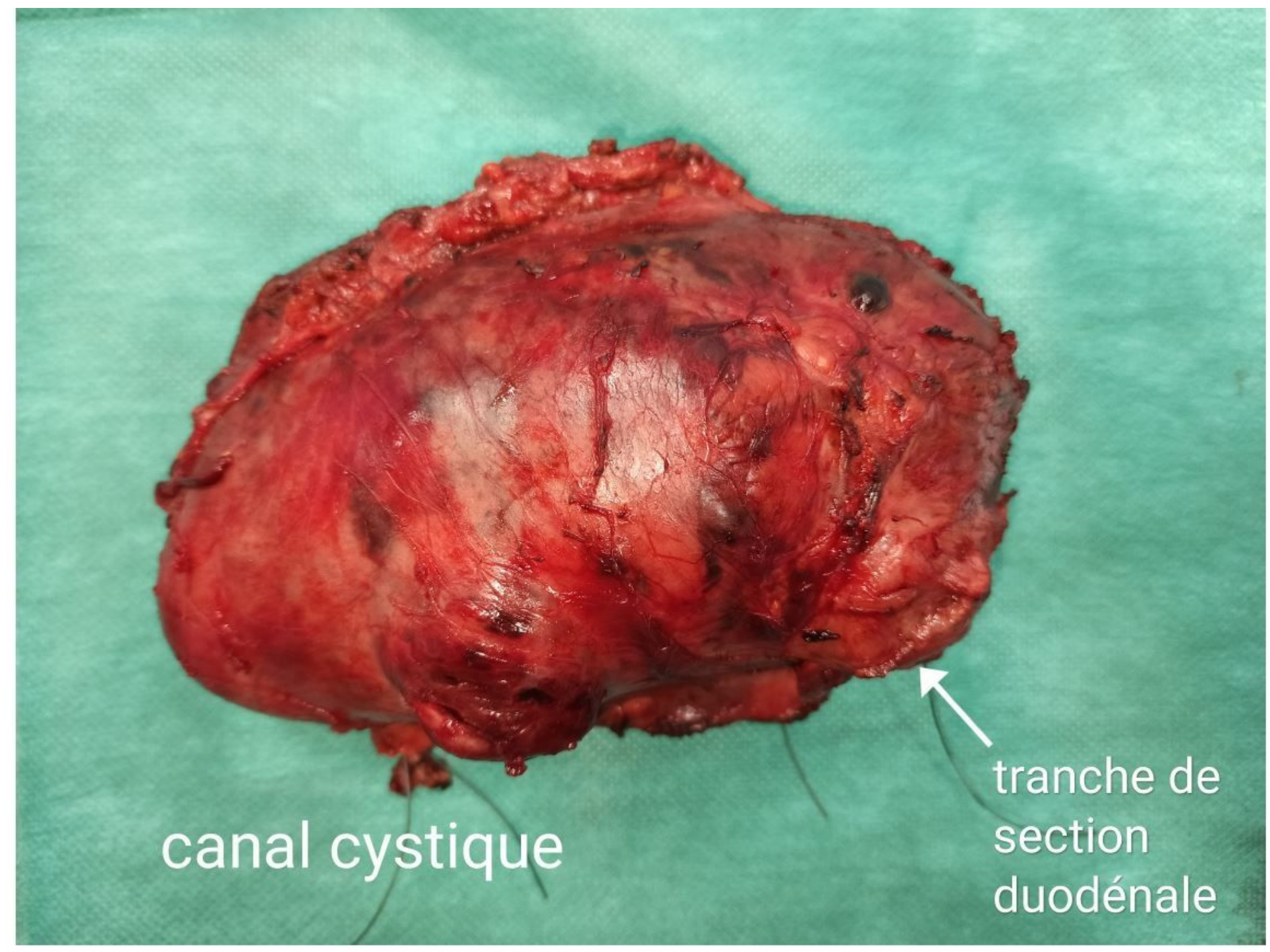

Figure 2

Image showing the post-operative resection patch 


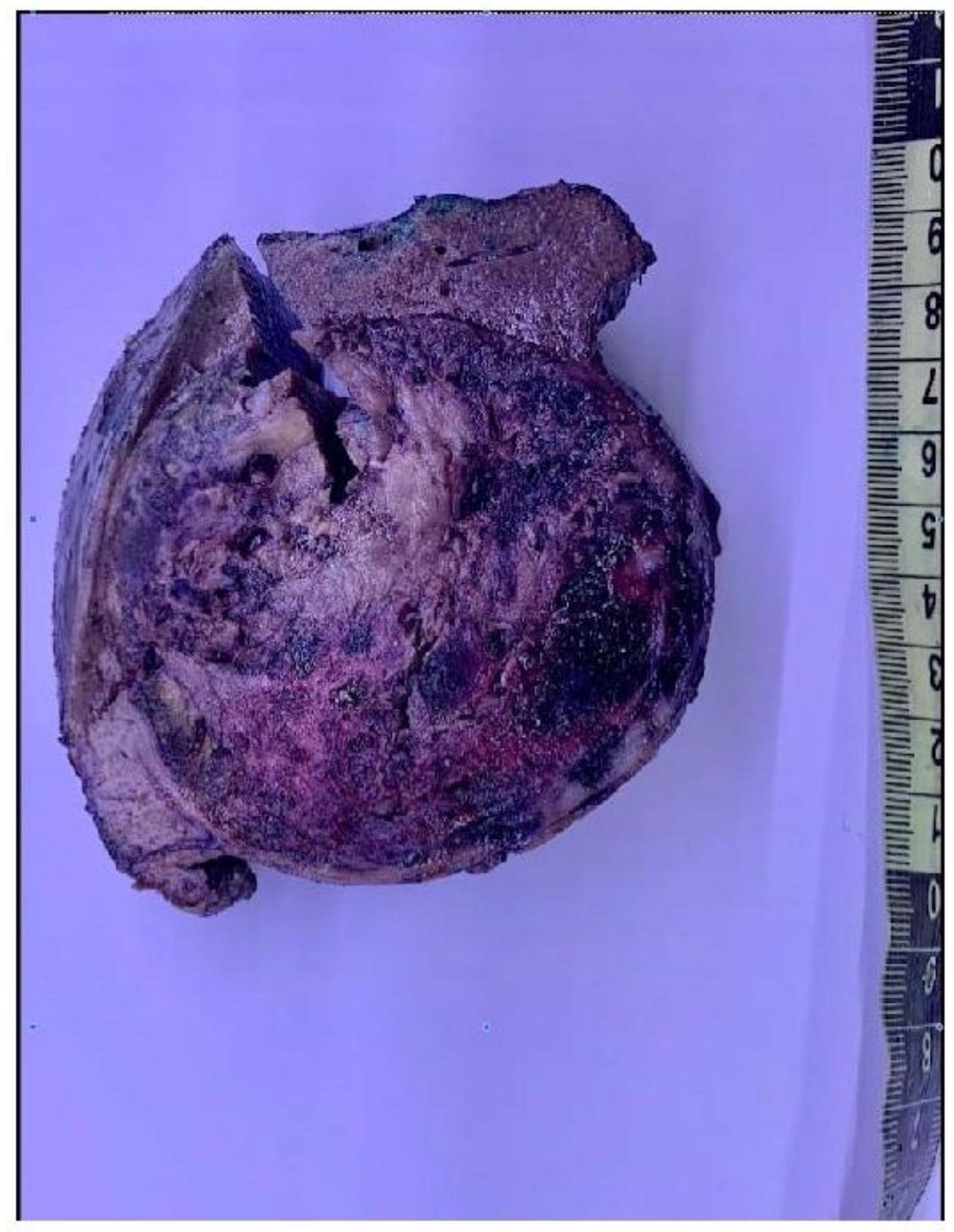

Figure 3

Image showing the appearance and infiltration of the tumor in the gallbladder, and a part of the liver segment 


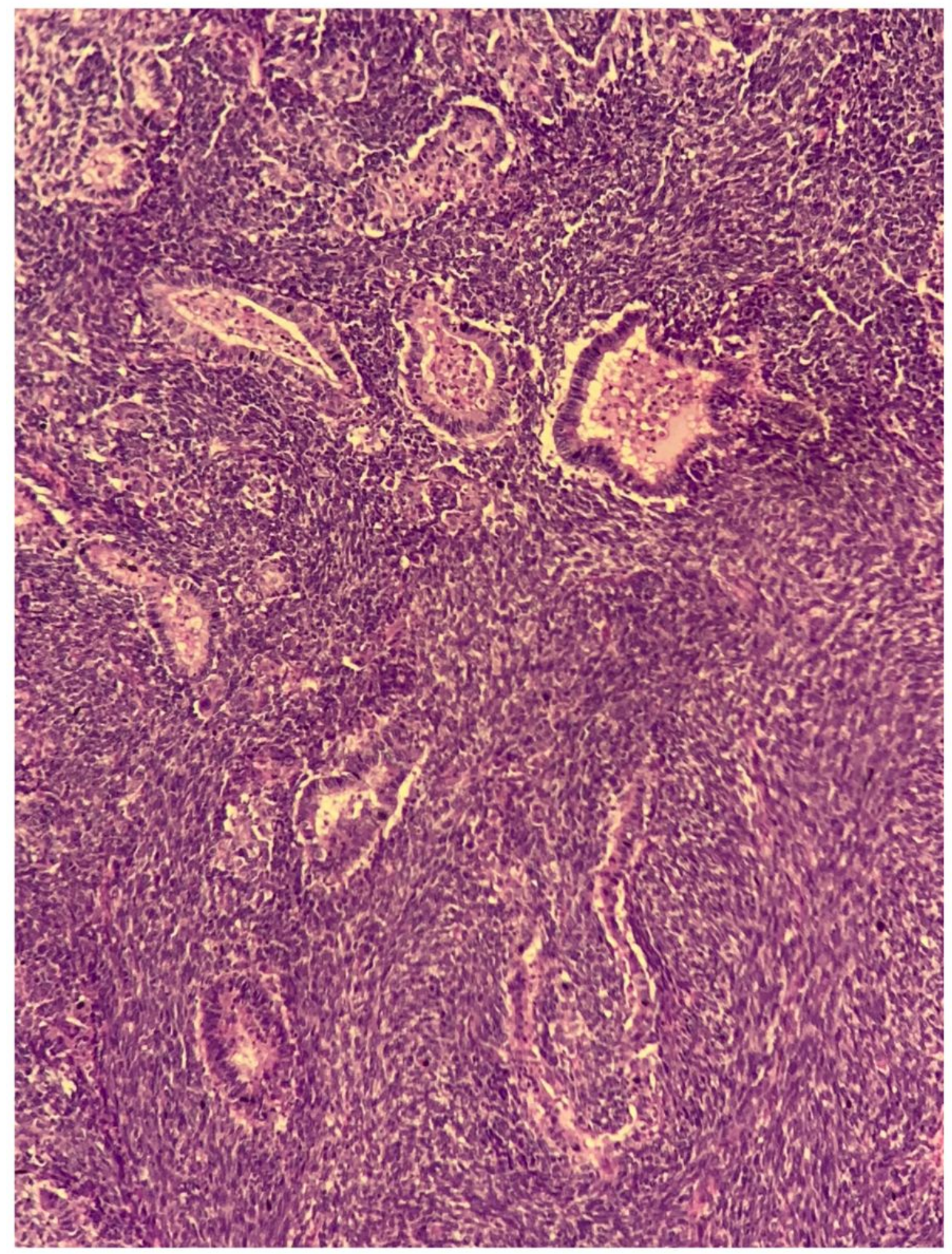

Figure 4

Image showing the presence of a mixed proliferation, carcinomatous represented by glands, and sarcomatous grouped in fascicles. 


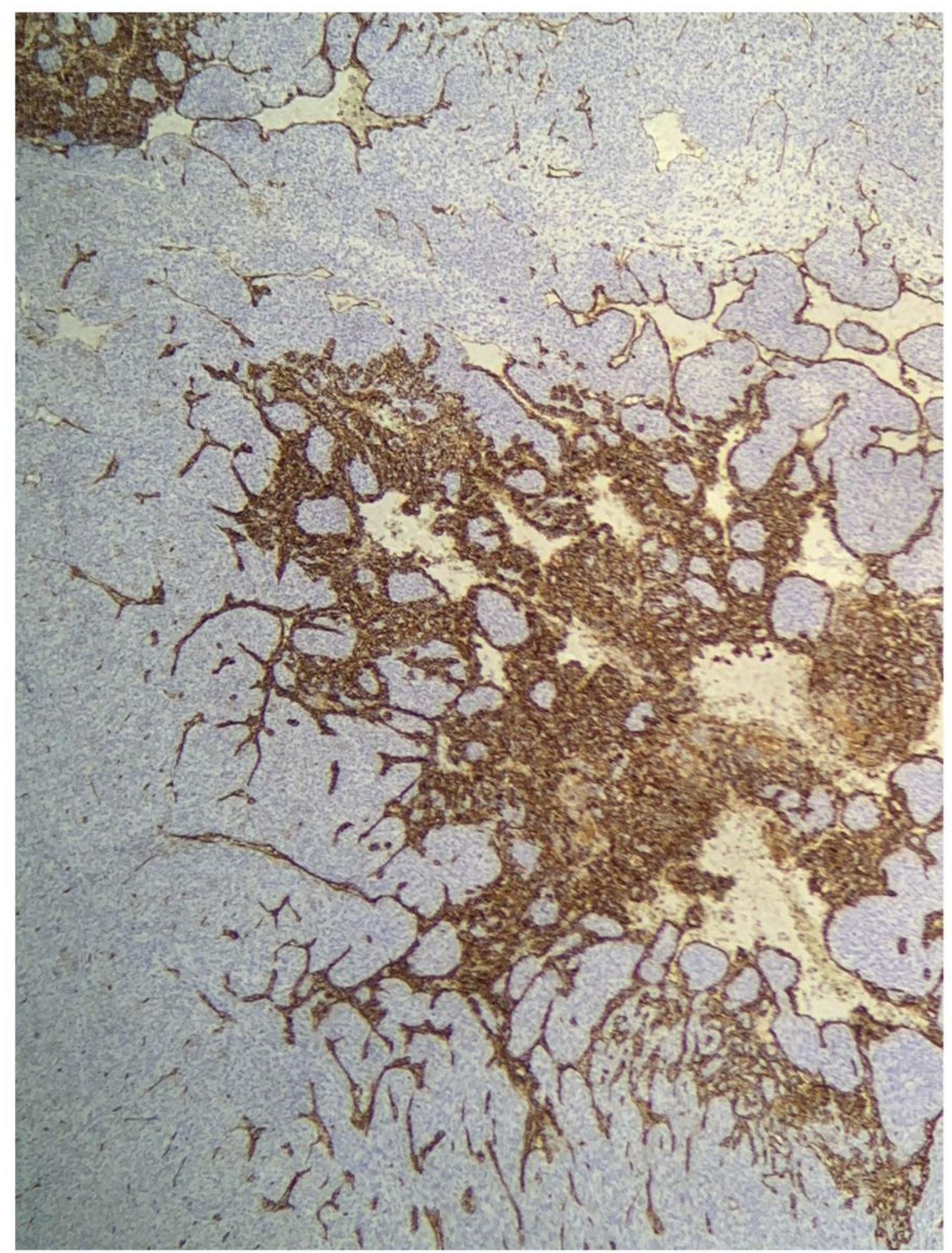

Figure 5

Pictures showing the positivity of tumor cells in areas of vascular differentiation by CD31. [x10] 


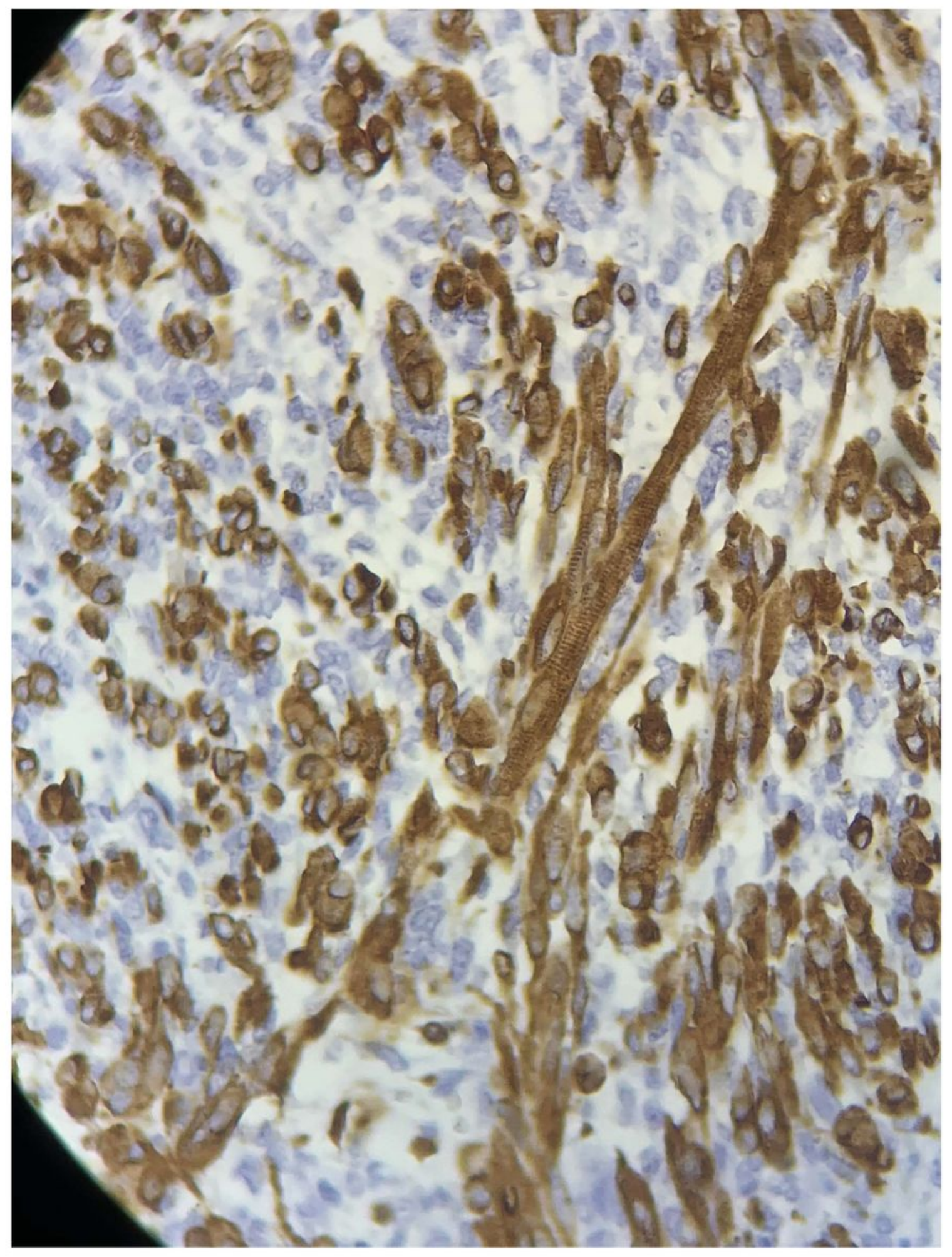

Figure 6

Picture showing the positivity of tumor cells in areas of striated muscle differentiation by Desmine . [x40] 


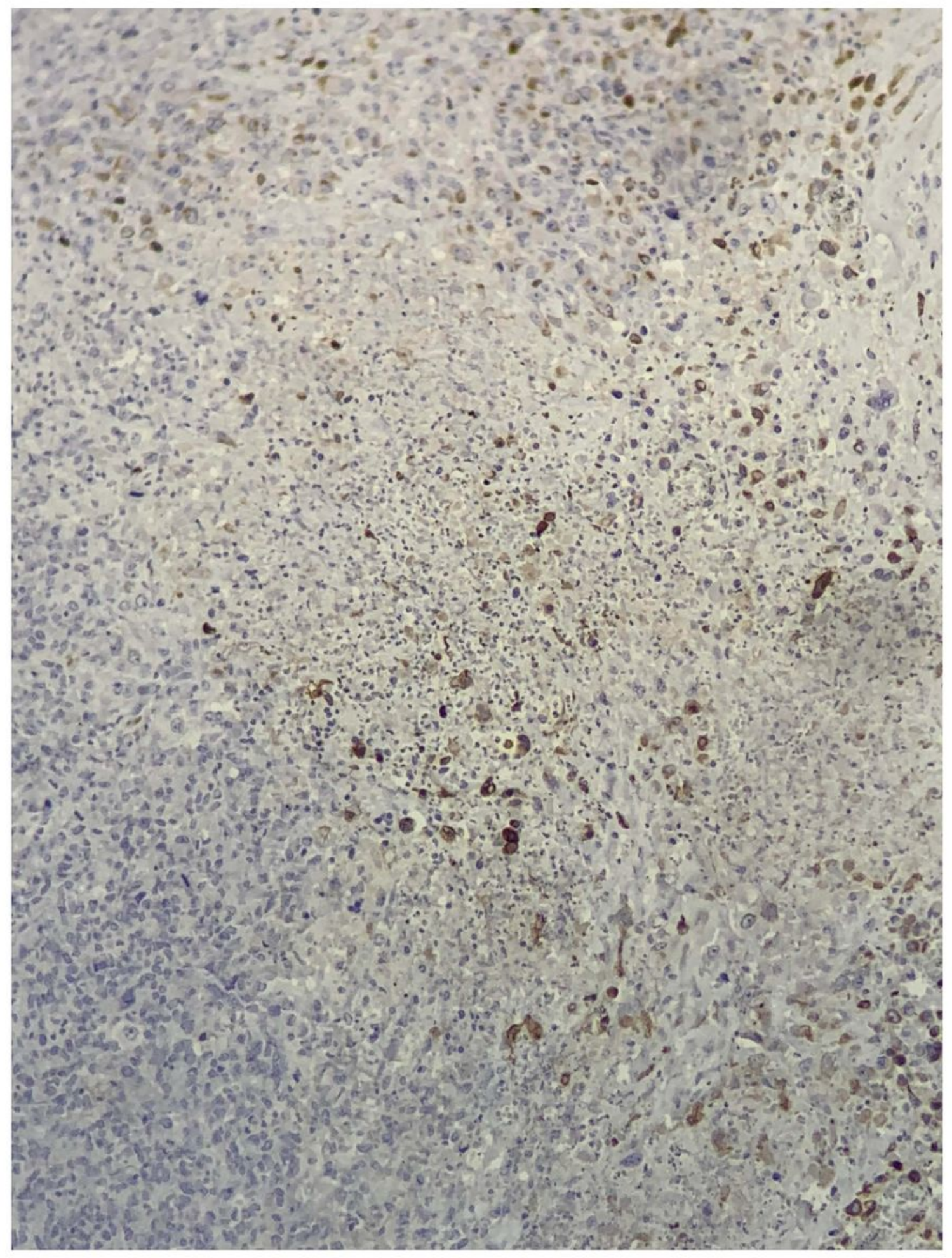

Figure 7

Image showing the focal positivity of tumor cells of the carcinomatous component by CK7. [x20] 